\title{
4D PET Scan
}

National Cancer Institute

\section{Source}

National Cancer Institute. 4D PET Scan. NCI Thesaurus. Code C125609.

Positron emission tomography imaging collected over time. It is designed to capture the internal movement of organs and the metabolism of the tumor. It creates the most complete and accurate information on the tumor and provides information how the tumor moves with breathing and other body motions. 